\title{
Randomized Controlled Trial of a Novel Silicone Device for the Packing of Cutaneous Abscesses in the Emergency Department: A Pilot Study
}

\author{
Aaron Brody' \\ John Gallien' \\ Brian Reed' \\ John Hennessy ${ }^{2}$ \\ Michael J Twiner ${ }^{1,3}$ \\ Jerry Marogil ${ }^{4}$
}

'Department of Emergency Medicine, Wayne State University, Detroit, MI, USA; ${ }^{2}$ College of Engineering, University of Michigan, Ann Arbor, MI, USA;

${ }^{3}$ Integrative Biosciences Center, Wayne State University, Detroit, MI, USA;

${ }^{4}$ Mar-Med Inc, Grand Rapids, MI, USA
Objective: Traditional treatment of abscesses in the emergency department includes packing with gauze; however, this can add pain and discomfort to the procedure and frequently involves a follow-up visit for packing removal. Alternatives to gauze packing have been proposed, but they may have disadvantages in the context of emergency care. The objective of this pilot study was to investigate the use of a novel silicone packing device - the DermaStent.

Methods: This was a randomized controlled pilot study of 50 patients from two urban emergency departments with uncomplicated superficial abscesses. The primary outcome was the likelihood of self-removal of packing gauze versus the silicone device. Secondary outcome measures included subjective clinician and patient metrics, such as pain and ease of use.

Results: Patients identified with simple cutaneous abscesses were randomized to intervention (packing with the novel silicone device, $n=25$ ) or standard care (gauze packing, $n=25$ ). Mean age was 36 years, 54\% were female, and 96\% identified as African American. Although it took longer to place, the silicone device ( 19.0 vs 15.3 minutes, $p=0.03$ ), pain scores were significantly lower $(4.3 / 10$ vs $7.1 / 10, p=0.008)$ and ease of use reported by physicians better in the silicone-device group $(4.8 / 5$ vs $4.0 / 5, p=0.002)$. A high unknown rate in the gauze-packing group limits discussion of the likelihood of selfremoval; however, the silicone device was more likely to remain in place $(60 \%)$ versus the gauze packing at 3 days $(24 \%, p=0.01)$.

Conclusion: This pilot randomized controlled trial compared the treatment of packing cutaneous abscesses with gauze versus using the silicone device. Limitations in the data prevent discussion on likelihood of self-removal. However, the silicone device was more likely to remain in place at day 3 follow-up and was equally effective to gauze packing in abscess reduction while also improving patient-reported pain scores. It did take longer to place the silicone device; however, physicians reported better ease of use and removal. This pilot study is encouraging for additional larger-scale trials that are required to further assess the utility of this device in the emergency department.

Keywords: abscess, Derma-Stent, silicone device, gauze, packing

\section{Introduction}

Cutaneous abscesses are an infectious condition that affects millions of people each year, and their incidence is on the rise. Between 1993 and 2005, the annual number of emergency department (ED) visits in the US for skin and soft-tissue
Correspondence: Michael J Twiner Department of Emergency Medicine, 420I St Antoine, UHC-6G, Detroit, MI, 4820I, USA

Tel + 3 3/3-745-3040

Email mtwiner@med.wayne.edu 
infections increased from 1.2 to 3.4 million, largely attributed to a rise in the prevalence of methicillin-resistant Staphylococcus aureus in the community. ${ }^{1,2}$

Although currently under debate, ${ }^{3,4}$ many providers believe that for uncomplicated cutaneous abscesses seen in the ED, the standard treatment is incision and drainage at the bedside. ${ }^{5}$ It is also not uncommon to insert a strip of gauze packing to maintain the open surgical incision, enhance drainage, and prevent reaccumulation of the abscess. If the abscess is packed, patients may be instructed to return to the ED in 2-3 days to have the packing removed. However, this gauze packing may not be effective, as it often falls out spontaneously and prematurely without providing any clinical benefit. ${ }^{6}$ Additionally, the standard packing procedure and its removal may be painful, due to the gauze hardening after absorbing purulent discharge and blood.

A more novel approach advocates for the use of a vessel loop, such as a silicone string loop, for purposes of packing and drainage. ${ }^{7-9}$ While this approach may reduce pain and may be superior in terms of healing time and treatment failure, particularly in children, ${ }^{10}$ it is not readily available in the ED or outpatient clinics. Stringloop devices are typically sterile silicone devices used in vascular procedures. However, for abscesses, the smaller incision needed might preclude irrigation of the abscess cavity, and the vessel loops may be susceptible to malfunction, since they typically require a surgical knot through two incisions.

The objective of the present study was to investigate the use of a novel silicone packing device, the Derma-Stent, which has been developed for use in the ED as a commercial device similar to the vessel loop. The device has multiple benefits due to its silicone design: it does not absorb bodily fluids, it increases patient compliance, and it decreases device failure, while achieving the objective of wound healing by maintaining the opening of the surgical incision(s). The first use of this device was described in a case report, and led to a successful clinical and cosmetic outcome. ${ }^{11}$ The device is similar to other loop devices that have resulted in lower failure rates in children, but otherwise equivocal rates in adults. ${ }^{10}$ In this paper, we describe a pilot randomized control trial (RCT) of the silicone device. Compared to standard gauze packing, we posit that this novel commercial device will facilitate self-removal, be better tolerated by patients, and be more likely to remain in the abscess cavity until proper removal.

\section{Methods}

We conducted a pilot RCT comparing the silicone device to traditional gauze packing in cutaneous abscesses of patients who presented to the ED. As a measurement of perceived agency over self-care, the primary outcome was the likelihood of self-removal of the silicone device versus gauze packing. Study participants were selected from consecutive patients presenting to either of two urban EDs in Detroit, MI, USA, with cutaneous abscesses large enough to require bedside incision, drainage, and packing, as determined by ED providers. Prospective participants were screened, consented, and enrolled by trained research associates 24 hours a day, 7 days a week between February 2017 and June 2018. Prior to randomization, abscesses had been measured with bedside ultrasound by trained personnel supervised by ultrasound fellowship-trained attending ED physicians. Inclusion criteria were age 18-90 years, total abscess dimensions $(x+y+z \mathrm{~cm})$ on ultrasound $\geq 5 \mathrm{~cm}$, assessment by an attending physician that the abscess would require incision, drainage, and packing, and informed consent. Exclusion criteria were patients requiring admission during initial ED visit, abscess drainage requiring procedural sedation or formal incision in the operating room, inability to comprehend consent or follow-up instructions, and prisoners.

Following consent and randomization, the treating ED physician sterilized the skin and anesthetized the abscess utilizing standard departmental protocols and supplies. The specific technique for localized anesthesia was at the discretion of the treating ED physician and not protocolized by the study; however, any patients receiving general anesthesia or systemic sedation were excluded from the study. All abscesses were irrigated, then packed using either an iodineimpregnated gauze strip (Curity Iodoform packing strip; Medtronic, Minneapolis, MN, USA) or the silicone device (Derma-Stent; Mar-Med, Grand Rapids, MI, USA). Figure 1 is a schematic illustration of the device and various configurations for its deployment, including single incision versus dual incision. In order to fit various sizes and shapes of abscess cavities, treating physicians can trim the body length or longitudinal arms of the device for insertion into one or two incisions. At physicians' discretion, the single- or double-incision technique can be employed. Provision of antibiotics was also at the discretion of the providers, but institutional guidelines strongly recommend antibiotics with methicillin-resistant Staphylococcus aureus coverage. 
1) Drain abscess using 1 or 2 incisions.

2) Measure the abscess along Derma-Stent. Cut to size.

3) Insert Derma-Stent into abscess cavity.

4) Remove Derma-Stent in accordance with clinician suggestions.

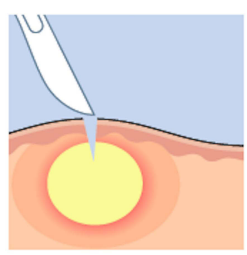

OR
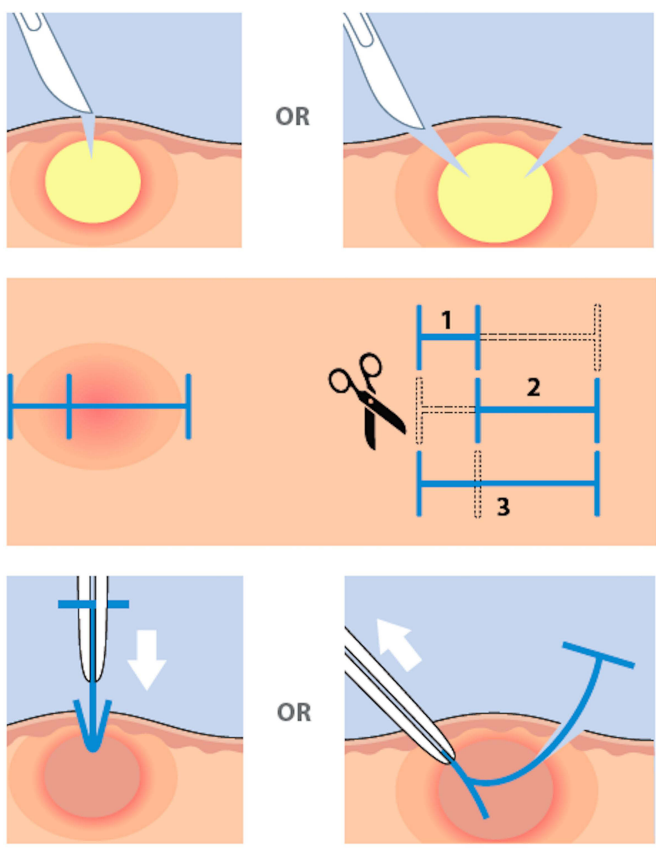

OR
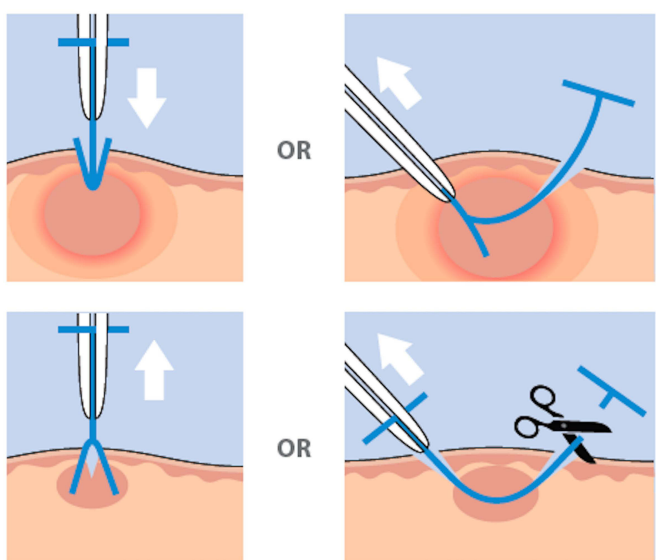

OR

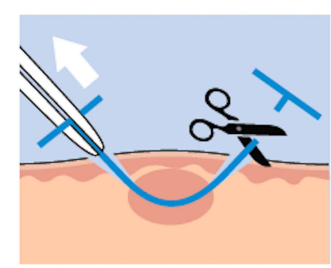

Figure I Schematic illustration of the Derma-Stent device and various configurations for deployment in the abscess cavity.

Enrolled patients were scheduled for follow-up on day 3 , during which they answered a number of survey questions. All packing gauze or devices remaining in abscess cavities were removed by the patients themselves or by the treating physician, and abscess-cavity size was measured again. We performed a chart review at day 30 to assess for treatment failures, defined as a need for reincision or hospitalization for antibiotic therapy.

The ability of the patients to remove the device was the primary outcome. Other outcome measures included clinical failure, defined by the presence of fever, an increase in maximal diameter of erythema, or worsening of wound swelling and tenderness, cosmetic result at follow-up visits, as measured subjectively by participants utilizing a $1-5$ Likert scale, pain during the procedure and packing removal measured using a $0-10$ visual analogue scale, and acceptability and satisfaction surveys by both providers and participants regarding different aspects of the procedure, such as pain, duration, and ability to maintain a sterile field.

Descriptive statistics were used for comparison with data, and are presented as means $\pm \mathrm{SD}$. For exploratory analysis, $95 \%$ CIs were utilized to evaluate effect size.
Categorical and continuous results were analyzed utilizing chi-squared test and $t$-test probabilities, respectively, with $p<0.05$ considered significant. Due to the pilot nature of this study, the effect size was unknown and formal power analysis could not be performed. As such, 25 patients per treatment group were selected. Study data were collected and managed using REDCap (Research Electronic Data Capture) electronic data-capture tools hosted at Wayne State University (Detroit, MI, USA). REDCap is a secure, web-based application designed to support data capture for research studies. ${ }^{12}$ Analysis was performed with SAS software (SAS Institute, Cary, NC, USA). The institutional review board of Wayne State University approved this human research study, which was performed in accordance with the Declaration of Helsinki. The study was registered on www.clintrials.gov (NCT03171714).

\section{Results}

Fifty subjects were recruited and randomized evenly between the investigational silicone device and gauze 


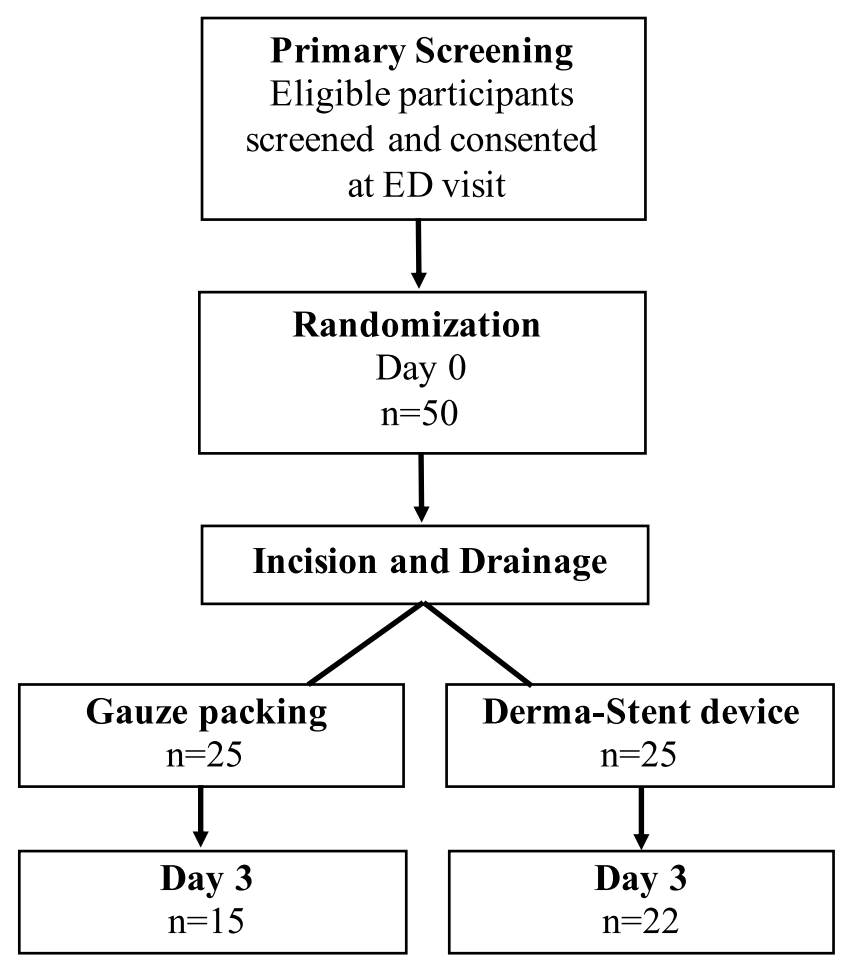

Figure 2 Consort diagram of enrolled patients.

packing. Mean age of the subjects was 36 years, $54 \%$ were female, and $96 \%$ identified as African American. Of the 25 patients in each group, 15 in the gauze-packing group and 22 in the silicone-device group were seen at day 3 follow-up appointments (Figure 2). Postrandomization baseline data are presented in Table 1. Most variables were similar between groups; however, there were more unemployed patients with a lower level of education in the siliconedevice group (Table 1). Average abscess size was similar between groups, though the procedure time for placement of the silicone device was almost 4 minutes longer (15.3 vs 19.0 minutes, $p=0.03$; Table 2). Perceived pain was significantly lower ( $4.3 / 10$ vs $7.1 / 10, p=0.008)$ and ease of use reported by the physician was better in the silicone-device group ( $p=0.002$ ).

At the day 3 follow-up appointment, abscess sizes had improved in both groups compared to day 0 , but no differences were detected between groups. Such measures as postinsertion pain and bleeding/discharge were not different, but ease of removal by the physician was improved in the silicone-device group $(p=0.011)$. Although there were a high number of unknown removals $(n=12)$ in the gauzepacking group, the silicone device was more likely to remain in place at the day 3 follow-up than the gauze packing ( $60 \%$ vs $24 \%$, respectively; $p=0.010)$.
There were no reported adverse outcomes during the initial 3 days of the study; however, 30-day chart review identified one patient with a history of HIV in the siliconedevice group that did not return for the day 3 follow-up appointment but rather came back to the ED around 2 weeks later and was admitted for intravenous antibiotics. The patient was not febrile or septic, and did not require reincision of the abscess.

\section{Discussion}

Cutaneous abscesses remain a common cause of ED visits, and there a variety of management options available. Although there is some debate about the utility of wound packing, ${ }^{3,4,6}$ many providers continue to use packing materials to facilitate ongoing drainage after initial incision and drainage, especially for abscesses $>5 \mathrm{~cm}$ in diameter or in patients who are at high risk of treatment failure. Because of the pain and discomfort associated with the use of traditional gauze packing, a more comfortable and secure alternative is desirable. The need for an effective packing material that can be well tolerated has led to improvised methods of packing from a variety of materials, as well as the development of novel silicone-packing solutions, ${ }^{10}$ including the silicone device. ${ }^{11}$

The silicone device was derived from the concept of a price-tag fastener. Its small longitudinal bars can maintain its position, and can thus be modified based on the size and shape of the abscess. While similar devices have not resulted in reduced failure rates in adults, they have been shown to be superior to gauze packing in children. ${ }^{10}$ In this pilot RCT, the ability of the patients to remove the silicone device versus gauze packing was our primary outcome. However, limitations in the study design and patient follow-up did not allow for commentary as intended. Alternatively, presence of the packing device in the abscess cavity at day 3 heavily favored the siliconepacking device, validating the functionality of the device design. Furthermore, the silicone device was shown to be equally effective as gauze packing in abscess reduction while improving patient-reported pain scores, despite the procedure taking 3.7 minutes longer. The physicians reported better ease of use and removal. Despite an unequal number of missing data regarding removal of the packing gauze, the silicone device appeared to remain in the abscess more effectively than gauze. Overall, with the lone exception of procedure time (which may improve with experience), the silicone device was superior or 
Table I Baseline variables for patients packed with gauze or silicone device

\begin{tabular}{|c|c|c|c|}
\hline & Gauze packing & Silicone device & \multirow[t]{2}{*}{$p$} \\
\hline & n/mean (\%/SD) & n/mean (\%/SD) & \\
\hline \multicolumn{4}{|l|}{ Demographics } \\
\hline Age (years) & $33(10)$ & $39(14)$ & 0.192 \\
\hline Female & $16(64)$ & II (44) & 0.156 \\
\hline Employed or student & $18(72)$ & $12(48)$ & 0.044 \\
\hline Insured & $22(88)$ & $23(92)$ & 0.672 \\
\hline \multicolumn{4}{|l|}{ Education (highest level attained) } \\
\hline Elementary/high school & $8(33)$ & $14(61)$ & 0.048 \\
\hline Associate degree or technical school & $3(12)$ & $2(9)$ & 0.664 \\
\hline College/university & $13(54)$ & $7(30)$ & 0.081 \\
\hline \multicolumn{4}{|l|}{ Identified ethnicity } \\
\hline African American & $24(96)$ & $24(96)$ & - \\
\hline Hispanic or Latino & I (4) & 0 & - \\
\hline Other & I (4) & I (4) & - \\
\hline \multicolumn{4}{|l|}{ Medical history } \\
\hline Diabetes (type 2) & $5(20)$ & $5(20)$ & - \\
\hline Hidradenitis suppurativa & $5(20)$ & $3(12.5)$ & 0.855 \\
\hline HIV & I (4) & $2(8)$ & - \\
\hline Hypertension & $6(24)$ & II (44) & 0.114 \\
\hline Immunosuppressive therapy & 0 & I (4) & - \\
\hline Congestive heart failure & 0 & I (4) & - \\
\hline \multicolumn{4}{|l|}{ Substances of abuse } \\
\hline Alcohol & $3(12)$ & $0(0)$ & 0.235 \\
\hline Tobacco & $18(72)$ & $22(88)$ & 0.157 \\
\hline Heroin & I (4) & $2(8)$ & - \\
\hline Cocaine & I (4) & $2(8)$ & - \\
\hline
\end{tabular}

Note: "-" represents no significant difference or insufficient data for analysis.

equivalent in terms of patients, physicians, and clinical outcomes.

There was one potential adverse event identified on 30day chart review. A patient in the silicone-device group with a history of HIV had a subsequent ED visit and admission for intravenous $\mathrm{V}$ antibiotics. As the patient did not return at day 3 , it could not be determined if the device played a role in treatment failure.

\section{Limitations}

As a pilot, this study was limited primarily by its small sample and discrepancy in follow-up rates for the gauzepacking group, which directly impacted our primary outcome. With 12 patients $(48 \%)$ categorized as "unknown removal", this limited our conclusions regarding this pilot trial. Additionally, another shortcoming was the lack of standardized protocols for the provision of antibiotics and detailed, protocolized techniques for the incision and drainage procedures, such as method of anesthesia, amount of irrigation, length of incision, and amount of packing in the gauze packing. Although this strategy confers the benefits of a pragmatic trial design, it limits precise comparisons and isolation of the intervention as an independent variable. Subsequent RCTs with this device should also expand their follow-up times to further elucidate any possible relationship between the silicone device and adverse outcomes, such as seen in one patient.

\section{Conclusion}

This pilot RCT compared the treatment of packing cutaneous abscesses with gauze versus using a silicone device. The silicone device was more likely to remain in place at day 3 follow-up, and was equally 
Table 2 Outcomes of patients randomized to abscesses packed with gauze or silicone device

\begin{tabular}{|c|c|c|c|c|c|}
\hline & \multicolumn{2}{|c|}{ Gauze packing } & \multicolumn{2}{|c|}{ Silicone device } & \multirow[t]{2}{*}{$p$} \\
\hline & Mean (SD) & $\mathbf{n}$ & Mean (SD) & $\mathbf{n}$ & \\
\hline \multicolumn{6}{|l|}{ Day 0} \\
\hline Abscess size $(\mathrm{cm}, x+y+z)$ & $6.5(1.6)$ & 25 & $6.8(1.6)$ & 25 & 0.678 \\
\hline Procedure time (minutes) & I5.3 (II.3) & 25 & $19.0(7.8)$ & 25 & 0.030 \\
\hline \multicolumn{6}{|l|}{ Patient-reported outcomes } \\
\hline Pain during procedure $(0-10)$ & $7.1(2.9)$ & 25 & $4.3(3.6)$ & 25 & 0.008 \\
\hline Experience as a whole $(\mathrm{I}-5)$ & $4.0(1.4)$ & 25 & $4.5(1.0)$ & 25 & 0.114 \\
\hline Satisfaction with time $(1-5)$ & $4.7(0.6)$ & 25 & $4.7(0.5)$ & 25 & 0.772 \\
\hline \multicolumn{6}{|l|}{ Physician-reported outcomes } \\
\hline Ease of use $(1-5)$ & $4.0(1.0)$ & 25 & $4.8(0.5)$ & 25 & 0.002 \\
\hline Ability to maintain sterile field $(\mathrm{I}-5)$ & $3.5(1.2)$ & 25 & $4(1.1)$ & 25 & 0.142 \\
\hline \multicolumn{6}{|l|}{ Day 3 follow-up } \\
\hline Abscess size $(\mathrm{cm}, x+y+z)$ & $3.6(1.4)$ & 14 & $3.6(1.5)$ & 21 & 0.884 \\
\hline Device/packing already removed by patient & & 7 & & 6 & 0.732 \\
\hline Device/packing removed by physician & & 6 & & 15 & 0.010 \\
\hline Unknown removal & & 12 & & 4 & 0.031 \\
\hline \multicolumn{6}{|l|}{ Patient-,reported outcomes } \\
\hline Pain since procedure $(0-10)$ & $4.2(2.7)$ & 14 & $3.4(2.8)$ & 21 & 0.246 \\
\hline Discharge and bleeding $(I-5)$ & $3.8(1.4)$ & 14 & $3.7(1.2)$ & 21 & 0.711 \\
\hline \multicolumn{6}{|l|}{ Physician-reported outcomes } \\
\hline Ease of removal $(I-5)$ & $4.8(0.4)$ & 10 & $4.8(0.5)$ & 19 & 0.570 \\
\hline Satisfaction with removal $(I-5)$ & $4.0(1.0)$ & 11 & $4.8(0.5)$ & 20 & 0.011 \\
\hline Appearance of scar (I-5) & $3.9(0.9)$ & 13 & $4.3(0.9)$ & 21 & 0.211 \\
\hline
\end{tabular}

Notes: Likert scale (I-5): I, strongly disagree; 2, disagree; 3, neutral; 4, agree; 5 strongly agree. Pain characterized by visual analogue scale: 0 , no pain; 5 , distressing pain; I0, unbearable pain. Values in bold indicate $p<0.05$.

effective as gauze packing in abscess reduction, while improving patient-reported pain scores. It did take longer to place the silicone device; however, physicians reported better ease of use and removal. These results are very promising as pilot data and provide justification for a larger, multicenter trial, but would likely need more standardized protocols for the incision and drainage procedures. Future models of the device could be modified and enlarged for use with abscesses drained in the operating room by surgeons.

\section{Data Sharing Statement}

Deidentified study-participant data will be made available for academic purposes upon request to the corresponding author. Data include recruitment tools, consent forms, and participant baseline and outcome data. All reasonable requests will be honored.

\section{Author Contributions}

All authors contributed to data analysis, drafting or revising the article, have agreed on the journal to which the article will be submitted, gave final approval to the version to be published, and agree to be accountable for all aspects of the work.

\section{Funding}

This work was made possible through a grant from the State of Michigan Corporate Relations Network, Small Company Innovation Program, and Mar-Med Inc.

\section{Disclosure}

$\mathrm{AB}$ received research funding and salary support from Mar-Med related to this project. JM is an employee and holds partial ownership of Mar-Med. All other authors report no conflicts of interest. 


\section{References}

1. Pallin DJ, Egan DJ, Pelletier AJ, Espinola JA, Hooper DC, Camargo CA. Increased US emergency department visits for skin and soft tissue infections, and changes in antibiotic choices, during the emergence of community-associated methicillin-resistant Staphylococcus aureus. Ann Emerg Med. 2008;51:291-298. doi:10.1016/j.annemergmed.2007.12.004

2. Qualls ML, Mooney MM, Camargo CA, Zucconi T, Hooper DC, Pallin DJ. Emergency department visit rates for abscess versus other skin infections during the emergence of community-associated methicillin-resistant Staphylococcus aureus, 1997-2007. Clin Infect Dis. 2012;55:103-105. doi:10.1093/cid/cis342

3. O'Malley GF, Dominici P, Giraldo P, et al. Routine packing of simple cutaneous abscesses is painful and probably unnecessary. Acad Emerg Med. 2009;16:470-473. doi:10.1111/j.1553-2712.2009.00409.x

4. Kessler DO, Krantz A, Mojica M. Randomized trial comparing wound packing to no wound packing following incision and drainage of superficial skin abscesses in the pediatric emergency department. Pediatr Emerg Care. 2012;28:514-517. doi:10.1097/PEC.0b013e $3182587 \mathrm{~b} 20$

5. Singer AJ, Talan DA. Management of skin abscesses in the era of methicillin-resistant Staphylococcus aureus. $N$ Engl $J$ Med. 2014;370:1039-1047. doi:10.1056/NEJMra1212788
6. List M, Headlee D, Kondratuk K. Treatment of skin abscesses: a Review of Wound Packing and Post-Procedural Antibiotics. S D Med. 2016;69:113-119.

7. Tsoraides SS, Pearl RH, Stanfill AB, Wallace LJ, Vegunta RK. Incision and loop drainage: a minimally invasive technique for subcutaneous abscess management in children. $J$ Pediatr Surg. 2010;45:606-609. doi:10.1016/j.jpedsurg.2009.06.013

8. Ladd AP, Levy MS, Quilty J. Minimally invasive technique in treatment of complex, subcutaneous abscesses in children. J Pediatr Surg. 2010;45:1562-1566. doi:10.1016/j.jpedsurg.2010.03.025

9. Gaszynski R, Punch G, Verschuer K. Loop and drain technique for subcutaneous abscess: a safe minimally invasive procedure in an adult population. ANZ J Surg. 2018;88(1-2):87-90.

10. Ladde J, Baker S, Lilburn N, Wan M, Papa L. A Randomized Controlled Trial of novel loop drainage technique versus standard incision and drainage in the treatment of skin abscesses. Acad Emerg Med. 2020;27:1229-1240. doi:10.1111/acem.14106

11. Brody AM, Gallien J, Murphy D, Marogil JA. A novel silicon device for the packing of cutaneous abscesses. $J$ Emerg Med. 2019;56:298-300. doi:10.1016/j.jemermed.2018.12.009

12. Harris PA, Taylor R, Thielke R, Payne J, Gonzalez N, Conde JG. Research electronic data capture (REDCap)-a metadata-driven methodology and workflow process for providing translational research informatics support. $J$ Biomed Inform. 2009;42:377-381. doi:10.1016/j.jbi.2008.08.010
Open Access Emergency Medicine

\section{Publish your work in this journal}

The Open Access Emergency Medicine is an international, peerreviewed, open access journal publishing original research, reports, editorials, reviews and commentaries on all aspects of emergency medicine. The manuscript management system is completely online and includes a very quick and fair peer-review system, which is all easy to use. Visit http://www.dovepress.com/testimonials.php to read real quotes from published authors. 